\title{
The Frank Stinchfield Award
}

\section{Total Hip Arthroplasty for Femoral Neck Fracture Is Not a Typical DRG 470: A Propensity-matched Cohort Study}

\author{
William W. Schairer MD, Joseph M. Lane MD, David A. Halsey MD, Richard Iorio MD, \\ Douglas E. Padgett MD, Alexander S. McLawhorn MD, MBA
}

Published online: 6 May 2016

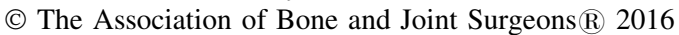

\begin{abstract}
Background Hip fractures are a major public health concern. For displaced femoral neck fractures, the needs for medical services during hospitalization and extending beyond hospital discharge after total hip arthroplasty (THA) may be different than the needs after THA performed for osteoarthritis (OA), yet these differences are largely uncharacterized, and the Medicare Severity Diagnosis-Related Groups system does not distinguish between THA performed for fracture and OA.

Questions/purposes (1) What are the differences in inhospital and 30-day postoperative clinical outcomes for THA performed for femoral neck fracture versus OA? (2) Is a patient's fracture status, that is whether or not a patient has a femoral neck fracture, associated with differences in in-hospital and 30-day postoperative clinical outcomes after THA?
\end{abstract}

One of the authors (JML) lists the following relevant financial activities outside of this work and/or any other relationships or activities that readers could perceive to have influenced, or that give the appearance of potentially influencing, this manuscript: Amgen, Inc (Thousand Oaks, CA, USA) in the amount of less than USD 10,000; Eli Lilly and Company (Indianapolis, IN, USA) in the amount of less than USD 10,000; Graftys (Aix-en-Provence, France) in the amount of less than USD 10,000; Kuros Biosurgery AG (Zürich, Switzerland) in the amount of less than USD 10,000; Bone Therapeutics (Brussels, Belgium) in the amount of less than USD 10,000; and Merck \& Co, Inc (Kenilworth, NJ, USA) in the amount of USD 100,001 to USD 1,000,000. One of the authors (DEP) lists the following relevant financial activities outside of this work and/or any other relationships or activities that readers could perceive to have influenced, or that give the appearance of potentially influencing, this manuscript: Mako Surgical Corp (Davie, FL, USA) in the amount of less than USD 10,001 to USD 100,000; and Stryker Corporation (Kalamazoo, MI, USA) in the amount of less than USD 10,000. All ICMJE Conflict of Interest Forms for authors and Clinical Orthopaedics and Related Research $\mathbb{\circledR}$ editors and board members are on file with the publication and can be viewed on request.
Methods The National Surgical Quality Improvement Program (NSQIP) database, which contains outcomes for surgical patients up to 30 days after discharge, was used to identify patients undergoing THA for OA and femoral neck fracture. OA and fracture cohorts were matched one-to-one using propensity scores based on age, gender, American Society of Anesthesiologists grade, and medical comorbidities. Propensity scores represented the conditional probabilities for each patient having a femoral neck fracture based on their individual characteristics, excluding their actual fracture status. Outcomes of interest included operative time, length of stay (LOS), complications, transfusion, discharge destination, and readmission. There were 42,692 patients identified (41,739 OA; 953 femoral neck fractures) with 953 patients in each group for the matched analysis.

Each author certifies that his or her institution approved or waived approval for the human protocol for this investigation and that all investigations were conducted in conformity with ethical principles of research.

This work was performed at the Hospital for Special Surgery, New York, NY, USA.

W. W. Schairer, J. M. Lane, D. E. Padgett,

A. S. McLawhorn ( $\square)$

Department of Orthopaedic Surgery, Hospital for Special

Surgery, 535 E 70th Street, New York, NY 10021, USA

e-mail: mclawhorna@hss.edu

D. A. Halsey

Department of Orthopaedic Surgery, University of Vermont

Medical College, South Burlington, VT, USA

R. Iorio

NYU Langone Medical Center, Hospital for Joint Diseases,

New York, NY, USA 
Results For patients with fracture, operative times were slightly longer (98 versus 92 minutes, $\mathrm{p}=0.015$ ), they experienced longer LOS ( 6 versus 4 days, $\mathrm{p}<0.001$ ), and the overall frequency of complications was greater compared with patients with OA $(16 \%$ versus $6 \%$, p < 0.001$)$. Although the frequency of preoperative transfusions was higher in the fracture group $(2.0 \%$ versus $0.2 \%$, $\mathrm{p}=0.002)$, the frequency of postoperative transfusion was not different between groups (27\% versus $24 \%$, $\mathrm{p}=0.157)$. Having a femoral neck fracture versus OA was strongly associated with any postoperative complication (odds ratio [OR], 2.8; 95\% confidence interval [CI], 2.13.8]; $\mathrm{p}<0.001$ ), unplanned readmission (OR, 1.8; 95\% CI, $1.0-3.2 ; \mathrm{p}=0.049)$, and discharge to an inpatient facility (OR, 1.7; 95\% CI, 1.4-2.0; $\mathrm{p}<0.001$ ).

Conclusions Compared with THA for OA, THA for femoral neck fracture is associated with greater rates of complications, longer LOS, more likely discharge to continued inpatient care, and higher rates of unplanned readmission. This implies higher resource utilization for patients with a fracture. These differences exist despite matching of other preoperative risk factors. As healthcare reimbursement moves toward bundled payment models, it would seem important to differentiate patients and procedures based on the resource utilization they represent to healthcare systems. These results show different expected resource utilization in these two fundamentally different groups of patients undergoing hip arthroplasty, suggesting a need to modify healthcare policy to maintain access to THA for all patients.

Level of Evidence Level III, therapeutic study.

\section{Introduction}

Fractures of the hip are a major public health concern. The annual incidence is approximately 1000 per 100,000 Medicare beneficiaries with 1-year mortality rates of nearly $30 \%$ [5, 16]. For femoral neck fractures, surgical treatment is nearly always considered in patients who are medically stable for intervention. Options include open or closed reduction and internal fixation, hemiarthroplasty, and THA. In elderly patients, the risk of nonunion after internal fixation is high after displaced fractures, and many studies have demonstrated improved hip function, better quality of life, and reduced frequencies of complications and re operations for both hemiarthroplasty [10] and THA [4, 8, 20] over internal fixation for these patients.

Good results with THA after femoral neck fractures were found over 20 years ago in more healthy and active patients [9], but hemiarthroplasty remained a mainstay treatment for elderly patients with these fractures. However, more recent evidence emphasizes the functional advantages of THA over hemiarthroplasty in the treatment of femoral neck fractures in active elderly patients [3, 11, 15, 21, 22]. Additionally, in patients who develop a painful hemiarthroplasty, conversion of hemiarthroplasty to THA is more difficult and associated with more complications than a primary THA for osteoarthritis (OA) [19]. Thus, in the current era, evidence strongly supports that in active adults with intact cognition who sustain a femoral neck fracture, THA is indicated rather than hemiarthroplasty.

Few studies have directly compared the outcomes after THA in patients with OA and patients with femoral neck fractures [1, 13, 14]. These studies have principally analyzed the in-hospital differences between these two groups of patients. They suggest that patients with femoral neck fractures are generally older than patients with OA, have more comorbidities, and are more likely to experience perioperative complications.

Hospitals, physicians, and other stakeholders providing care to patients undergoing THA largely have been reimbursed separately for their individual services under feefor-service payment models. Therefore, few financial incentives existed to better define the differences between THAs performed for fractures and those performed for OA. However, alternative reimbursement models such as bundled payments seek to improve coordination of care and reduce costs by paying one target price for the entire complement of services these providers supply to patients undergoing THA from the initial hospitalization through a predefined postoperative period, typically lasting between 30 and 90 days. These services over the specified period of time define an episode of care. Episodes of care are frequently linked to Medicare Severity Diagnosis-Related Groups (MS-DRG) codes 469 (Major joint replacement or reattachment of lower extremity with major complications or comorbidities) and 470 (Major joint replacement or reattachment of lower extremity without major complications or comorbidities) [7]. However, the MS-DRG system, under which hospitals have historically received re imbursement for care, has not distinguished between THA performed for fracture and THA performed for OA. Rather, this system has simply used International Classification of Diseases, 9th Revision (ICD-9) procedure codes or Current Procedural Terminology (CPT) procedure codes (and will use ICD-10 procedure codes) to identify hospitalizations reimbursed under MS-DRG 469 and 470. None of these procedure codes indicates the diagnoses for which THAs were performed.

As healthcare reimbursement moves toward bundled payment models, it would seem important to differentiate more explicitly and risk-stratify patients and procedures based on the expected resource utilization they represent to healthcare systems over longer periods of care than the 
hospital admission. We therefore sought to compare the perioperative and 30-day postoperative clinical outcomes of THA in patients with either a femoral neck fracture or $\mathrm{OA}$ and to determine the association between a patient's fracture status, that is whether or not a patient has a femoral neck fracture, and these clinical outcomes.

Specifically, we asked: (1) What are the differences in in-hospital and 30-day postoperative clinical outcomes for THA performed for femoral neck fracture versus OA? (2) Is a patient's fracture status associated with differences in in-hospital and 30-day postoperative clinical outcomes after THA?

\section{Patients and Methods}

This retrospective study used the National Surgical Quality Improvement Program (NSQIP) database, which is maintained by the American College of Surgeons. The NSQIP contains clinical data of surgical patients from hundreds of participating hospitals. Data are collected by trained and audited surgical clinical reviewers through chart review using rigorous data definitions. Perioperative data include diagnosis, procedure, operative time, estimated blood loss, and laboratory results. Patients are then followed for 30 days postoperatively to identify complications, readmissions, or reoperations. Demographic characteristics and medical comorbidities are also included. Recent audits have shown that the average interrater disagreement for the 135 variables the NSQIP collects is less than $2 \%$ [18].

The NSQIP data set from 2007 to 2013 was used to identify all patients who underwent primary THA (CPT procedure code 27130). Patients were included if they had either a primary diagnosis of femoral neck fracture (ICD-9 diagnosis codes $820.0 \times$ or 820.8 ) or OA (ICD-9 715.15, $725.15,735.25$, or 795.15); patients without one of these two diagnoses were excluded. Additional exclusion criteria included primary diagnoses of infection or cancer, because these were not relevant to the comparison of interest. Furthermore, patients with a primary diagnosis of implant complication were also excluded, because their cases were unlikely to represent primary procedures.

The primary outcome was the overall frequency of complications, defined by the NSQIP database, during the index hospitalization and the 30-day period after THA. Complications were further categorized and analyzed as either surgical or medical in nature. Secondary outcomes included reoperation or unplanned hospital readmission within 30 days, length of stay (LOS), transfusion requirement, and discharge destination. Multivariable statistical models were used to assess the associations between patient fracture status and differences in in-hospital and 30day postoperative clinical outcomes after THA.

\section{Statistical Analysis}

Patient demographics were compared between the femoral neck fracture and OA groups for both the entire cohorts as well as one-to-one matched groups using a propensity score. The technique of propensity score matching attempts to adjust for differences in measured baseline characteristics between the two groups. For this study, the propensity score was defined as the conditional probability of a patient with a femoral neck fracture given his or her baseline characteristics, including: age, gender, American Society of Anesthesiologists (ASA) score, medical comorbidities, and obesity (divided as Classes 1, 2, and 3). Although the NSQIP database contains information on preoperative laboratory values, these were not used for matching, because some variables had less than $80 \%$ coding and their inclusion would have resulted in reduced sample size and potential bias. A one-to-one match was performed using the nearest-neighbor propensity score [6]. This matching created an equal balance of characteristics between femoral neck fracture and OA patient groups.

Comparisons were performed using a chi-square test for categorical outcomes and a t-test for continuous outcomes. Multiple logistic regression was used to calculate the likelihood of complications, reported as odds ratios (ORs). The matched cohorts were used to compare the in-hospital and 30-day postoperative outcomes of interest. Statistical analyses were performed using STATA software (Version 12.1; Statacorp, College Station, TX, USA). Significance was set at a $\mathrm{p}$ value of $<0.05$.

A total of 42,692 patients who underwent THA were included; 41,739 patients were treated for OA and 953 patients were treated for a femoral neck fracture. After applying the propensity score matching, there were 953 patients in each group for a total of 1906 patients for the matched comparison. Before matching, the patients with femoral neck fracture were more likely to be white, female, older, and have a higher ASA grade with more medical comorbidities than patients with OA (Table 1). However, after matching, these differences were no longer significant, indicating a successful match on the desired covariates. There remained some differences in preoperative laboratory values after matching. The mean time between admission and the operating room was 0 days for patients with $\mathrm{OA}$ and 2 days for patients with fracture $(\mathrm{p}<0.001)$. Furthermore, $78 \%$ patients with fractures receive general anesthesia compared with $64 \%$ of patients in the OA group. The mean operative time was slightly 
Table 1. Patient demographics

\begin{tabular}{|c|c|c|c|c|c|c|}
\hline \multirow[t]{2}{*}{ Demographic data } & \multicolumn{3}{|c|}{ Unadjusted cohort comparison } & \multicolumn{3}{|c|}{ Matched cohort comparison } \\
\hline & $\mathrm{OA}$ & FNF & $\mathrm{p}$ value & $\mathrm{OA}$ & FNF & $\mathrm{p}$ value \\
\hline Number & 41,739 & 953 & - & 953 & 953 & - \\
\hline Age (years) & 65 & 73.4 & $<0.001 *$ & 73 & 73 & 0.748 \\
\hline Female & $55 \%$ & $68.3 \%$ & $<0.001 *$ & $70 \%$ & $68 \%$ & 0.428 \\
\hline \multicolumn{7}{|l|}{ Medical comorbidities } \\
\hline \multicolumn{7}{|l|}{ ASA } \\
\hline 1 & $4 \%$ & $3 \%$ & $0.041^{*}$ & $3 \%$ & $3 \%$ & 0.782 \\
\hline 2 & $56 \%$ & $32 \%$ & $<0.001 *$ & $32 \%$ & $32 \%$ & 0.883 \\
\hline 3 & $39 \%$ & $54 \%$ & $<0.001 *$ & $54 \%$ & $54 \%$ & 0.890 \\
\hline 4 & $2 \%$ & $11 \%$ & $<0.001 *$ & $11 \%$ & $11 \%$ & 0.885 \\
\hline Number of comorbidities & 1 & 2 & $<0.001 *$ & 2 & 2 & 0.332 \\
\hline Obesity BMI $30-34.9 \mathrm{~kg} / \mathrm{m}^{2}$ & $25 \%$ & $11 \%$ & $<0.001 *$ & $11 \%$ & $11 \%$ & 0.885 \\
\hline Obesity BMI $35-39.9 \mathrm{~kg} / \mathrm{m}^{2}$ & $12 \%$ & $3 \%$ & $<0.001 *$ & $3 \%$ & $3 \%$ & 1.000 \\
\hline Obesity BMI $\geq 40 \mathrm{~kg} / \mathrm{m}^{2}$ & $8 \%$ & $7 \%$ & 0.580 & $6 \%$ & $7 \%$ & 0.166 \\
\hline $\mathrm{CHF}$ & $0 \%$ & $2 \%$ & $<0.001 *$ & $3 \%$ & $2 \%$ & 0.452 \\
\hline Myocardial infarction & $0 \%$ & $1 \%$ & $<0.001 *$ & $0 \%$ & $1 \%$ & 0.483 \\
\hline Cardiac surgery & $1 \%$ & $2 \%$ & 0.003 & $2 \%$ & $2 \%$ & 0.248 \\
\hline PCI & $2 \%$ & $2 \%$ & 0.081 & $2 \%$ & $2 \%$ & 0.328 \\
\hline Angina & $0 \%$ & $0 \%$ & 0.961 & $0 \%$ & $0 \%$ & 0.571 \\
\hline COPD & $4 \%$ & $9 \%$ & $<0.001 *$ & $8 \%$ & $9 \%$ & 0.743 \\
\hline Renal failure & $0 \%$ & $0 \%$ & 0.416 & $0 \%$ & $0 \%$ & 0.571 \\
\hline Alcohol use & $1 \%$ & $1 \%$ & 0.564 & $1 \%$ & $1 \%$ & 0.796 \\
\hline PVD & $0 \%$ & $0 \%$ & 0.263 & $0 \%$ & $0 \%$ & 0.214 \\
\hline Dialysis & $0 \%$ & $1 \%$ & $<0.001$ & $1 \%$ & $1 \%$ & 0.834 \\
\hline Stroke—with deficit & $0 \%$ & $1 \%$ & $<0.001 *$ & $1 \%$ & $1 \%$ & 0.512 \\
\hline Stroke-no deficit & $0 \%$ & $2 \%$ & $<0.001 *$ & $2 \%$ & $2 \%$ & 0.871 \\
\hline Metastatic cancer & $0 \%$ & $1 \%$ & $<0.001 *$ & $1 \%$ & $1 \%$ & 0.277 \\
\hline Steroid use & $3 \%$ & $8 \%$ & $<0.001 *$ & $6 \%$ & $8 \%$ & 0.155 \\
\hline Weight loss & $0 \%$ & $1 \%$ & $<0.001 *$ & $0 \%$ & $1 \%$ & 0.176 \\
\hline Bleeding disorder & $3 \%$ & $14 \%$ & $<0.001 *$ & $15 \%$ & $4 \%$ & 0.435 \\
\hline Recent operation & $0 \%$ & $0 \%$ & 0.079 & $0 \%$ & $0 \%$ & 0.571 \\
\hline Hypertension & $58 \%$ & $59 \%$ & 0.412 & $59 \%$ & $59 \%$ & 1.000 \\
\hline TIA & $1 \%$ & $4 \%$ & $<0.001 *$ & $4 \%$ & $4 \%$ & 0.727 \\
\hline Smoking & $13 \%$ & $15 \%$ & 0.272 & $15 \%$ & $15 \%$ & 0.652 \\
\hline Diabetes & $11 \%$ & $15 \%$ & $<0.001 *$ & $14 \%$ & $15 \%$ & 0.362 \\
\hline \multicolumn{7}{|l|}{ Race } \\
\hline White & $81 \%$ & $84 \%$ & $0.007 *$ & $83 \%$ & $84 \%$ & 0.577 \\
\hline Black & $6 \%$ & $5 \%$ & 0.063 & $6 \%$ & $5 \%$ & 0.470 \\
\hline Asian & $1 \%$ & $2 \%$ & 0.145 & $2 \%$ & $2 \%$ & 0.737 \\
\hline Other & $12 \%$ & $9 \%$ & $0.013 *$ & $9 \%$ & $9 \%$ & 0.752 \\
\hline \multicolumn{7}{|l|}{ Preoperative laboratory values } \\
\hline Creatinine (mg/dL) & 0.9 & 1.0 & $0.004 *$ & 1.0 & 1.0 & 0.576 \\
\hline Albumin $(\mathrm{g} / \mathrm{dL})$ & 4.1 & 3.6 & $<0.001 *$ & 4.0 & 3.6 & $<0.001^{*}$ \\
\hline WBC count $\left(10^{9}\right.$ cells/L) & 7 & 10 & $<0.001^{*}$ & 7 & 10 & $<0.001 *$ \\
\hline Hematocrit (\%) & 41 & 37 & $<0.001 *$ & 39 & 37 & $<0.001 *$ \\
\hline Platelets $\left(10^{9} / \mathrm{L}\right)$ & 248 & 215 & $<0.001^{*}$ & 243 & 215 & $<0.001^{*}$ \\
\hline INR & 1.0 & 1.1 & $<0.001 *$ & 1.1 & 1.1 & 0.022 \\
\hline BUN (mg/dL) & 18 & 19 & $0.001 *$ & 20 & 19 & 0.076 \\
\hline
\end{tabular}


Table 1. continued

\begin{tabular}{|c|c|c|c|c|c|c|}
\hline \multirow[t]{2}{*}{ Demographic data } & \multicolumn{3}{|c|}{ Unadjusted cohort comparison } & \multicolumn{3}{|c|}{ Matched cohort comparison } \\
\hline & $\mathrm{OA}$ & FNF & $\mathrm{p}$ value & $\mathrm{OA}$ & FNF & $\mathrm{p}$ value \\
\hline Time to OR (days) & 0 & 2 & $<0.001 *$ & 0 & 2 & $<0.001 *$ \\
\hline \multicolumn{7}{|l|}{ Intraoperative data } \\
\hline \multicolumn{7}{|l|}{ Anesthesia } \\
\hline General & $61 \%$ & $78 \%$ & $<0.001 *$ & $62 \%$ & $78 \%$ & $<0.001 *$ \\
\hline Spinal & $39 \%$ & $22 \%$ & $<0.001 *$ & $38 \%$ & $22 \%$ & $<0.001 *$ \\
\hline Operative time (minutes) & 95 & 98 & $0.018 *$ & 92 & 98 & $0.015^{*}$ \\
\hline
\end{tabular}

* Probability values $<0.05 ; \mathrm{OA}=$ osteoarthritis; FNF = femoral neck fracture; ASA = American Society of Anesthesiologists physical status; $\mathrm{BMI}=$ body mass index $\mathrm{CHF}=$ congestive heart failure; $\mathrm{PCI}=$ percutaneous coronary intervention; $\mathrm{COPD}=$ chronic obstructive pulmonary disease; PVD = peripheral vascular disease; TIA $=$ transient ischemic attack; WBC $=$ white blood cell; INR $=$ international normalized ratio; $\mathrm{BUN}=$ blood urea nitrogen; $\mathrm{OR}=$ operating room.

longer for the fracture group than the OA group (98 versus 92 minutes, respectively; $\mathrm{p}=0.015$ ).

\section{Results}

There were differences in the in-hospital and 30-day postoperative clinical outcomes for THA performed for femoral neck fracture versus OA. Patients with fracture more frequently received preoperative transfusions compared with the OA group (2\% [19 of 953] versus $0.2 \%$ [two of 953], respectively; $\mathrm{p}<0.001$ ), although the frequency of postoperative transfusions was not different between the fracture cohort and the OA cohort (27\% [259 of 953] versus $24 \%$ [232 of 953], respectively; $p=0.157$ ). Mean hospital LOS was 4 days for patients with OA versus 6 days for patients with fracture $(p<0.001)$. The frequencies of patients experiencing in-hospital and 30-day postoperative complications were generally higher in the fracture group compared with the OA group (Table 2).

Logistic regression models illustrated the magnitude of the associations between the diagnosis of femoral neck fracture and numerous in-hospital and 30-day postoperative adverse outcomes (Table 2). Of note, the odds of mortality in the fracture group was 9.59 times that of the OA group (95\% confidence interval [CI], 2.90-31.64; $\mathrm{p}<0.001$ ). The odds of any postoperative complication in the fracture group was 2.80 times the OA group (95\% CI, 2.05-3.84; $\mathrm{p}<0.001)$. The fracture group was more likely to experience surgical complications (OR, 2.04; 95\% CI, 1.133.67; $\mathrm{p}=0.018)$; reoperations in particular were more likely in the fracture group (OR, 2.53; 95\% CI, 1.11-5.78; $\mathrm{p}=0.028$ ). Medical complications were also more likely in the fracture group compared with the OA group (OR, 3.13; 95\% CI, 2.20-4.45; $\mathrm{p}<0.001)$. Patients treated with THA for femoral neck fracture had greater risks for ventilator dependency over 48 hours (OR, 10.10; 95\% CI,
1.29-79.03; $\mathrm{p}=0.028)$, pneumonia (OR, 6.13; 95\% CI, 2.12-17.73; $\mathrm{p}<0.001)$, cardiac arrest (OR, 5.04; 95\% CI, 1.10-23.08; $\mathrm{p}=0.037$ ), unplanned intubation (OR, 3.44; 95\% CI, 1.27-9.37; $\mathrm{p}=0.016$ ), sepsis (OR, 4.38; 95\% CI, $1.24-15.42 ; \mathrm{p}=0.021)$, and urinary tract infection (OR, $2.10 ; 95 \% \mathrm{CI}, 1.26-3.48 ; \mathrm{p}=0.004)$ than patients treated for OA had. Hospital readmission was more likely in the fracture cohort (OR, 1.80; 95\% CI, 1.00-3.24; $\mathrm{p}=0.049$ ), and the fracture cohort was also more likely to be discharged to an inpatient facility compared with patients with OA (OR, 1.68; 95\% CI, 1.39-2.02; p < 0.001).

\section{Discussion}

There is much evidence supporting THA for treatment of femoral neck fractures in active elderly patients $[3,11,15$, $21,22]$. However, we found that patients undergoing THA for femoral neck fracture were more likely to die within 30 days of surgery, experience a major postoperative medical complication, have a reoperation, stay in the hospital longer, and be discharged to a postacute care facility than were patients undergoing THA for OA. Importantly, we observed these differences in a propensityscored matched cohort analysis that controlled for age, gender, and medical comorbidities. These results underscore that THA for fracture and THA for arthritis represent fundamentally different risks for adverse patient outcomes and hospital resource utilization. As hospitals assume increasingly more responsibility for the entire episode of care of these patients within bundled payment reimbursement models, it would seem important to more explicitly differentiate and risk-stratify patients and procedures based on the expected resource utilization they represent to healthcare systems.

There are several limitations to consider in this study. First, the data rely on records from a database, which may 
Table 2. Frequencies and odds for postoperative complications

\begin{tabular}{|c|c|c|c|c|}
\hline \multirow[t]{2}{*}{ Outcome } & \multicolumn{4}{|c|}{ Matched cohort comparison } \\
\hline & $\mathrm{OA}$ & FNF & OR $(95 \% \mathrm{CI})$ & $\mathrm{p}$ value \\
\hline Complication (overall) & $6 \%$ & $16 \%$ & $2.80(2.05-3.84)$ & $<0.001 *$ \\
\hline Complication (surgical) & $2 \%$ & $4 \%$ & $2.04(1.13-3.67)$ & $0.018 *$ \\
\hline Infection-superficial & $1 \%$ & $1 \%$ & $1.10(0.47-2.61)$ & 0.826 \\
\hline Infection-deep & $0.4 \%$ & $0.5 \%$ & $1.25(0.33-4.67)$ & 0.739 \\
\hline Wound dehiscence & $0.1 \%$ & $0.2 \%$ & $2.00(0.18-22.12)$ & 0.571 \\
\hline Reoperation & $1 \%$ & $3 \%$ & $2.53(1.11-5.78)$ & $0.028 *$ \\
\hline Complication (medical) & $5 \%$ & $13 \%$ & $3.13(2.20-4.45)$ & $<0.001^{*}$ \\
\hline Pneumonia & $0 \%$ & $3 \%$ & $6.13(2.12-17.73)$ & $<0.001 *$ \\
\hline Unplanned intubation & $0 \%$ & $2 \%$ & 3.44 (1.27-9.37) & $0.016^{*}$ \\
\hline DVT & $0 \%$ & $1 \%$ & $4.53(0.98-21.04)$ & 0.054 \\
\hline $\mathrm{PE}$ & $0 \%$ & $1 \%$ & $4.53(0.98-21.04)$ & 0.054 \\
\hline Ventilator over 48 hours & $0 \%$ & $1 \%$ & $10.10(1.29-79.03)$ & $0.028^{*}$ \\
\hline Renal insufficiency & $0 \%$ & $1 \%$ & $5.02(0.59-43.06)$ & 0.141 \\
\hline Acute renal failure & $0.0 \%$ & $0.1 \%$ & $1.73(0.11-27.68)$ & 0.700 \\
\hline Urinary tract infection & $2 \%$ & $5 \%$ & $2.10(1.26-3.48)$ & $0.004 *$ \\
\hline Stroke & $0 \%$ & $1 \%$ & $3.52(0.73-16.98)$ & 0.117 \\
\hline Cardiac arrest & $0 \%$ & $1 \%$ & $5.04(1.10-23.08)$ & $0.037 *$ \\
\hline Myocardial infarction & $1 \%$ & $1 \%$ & $1.50(0.53-4.24)$ & 0.440 \\
\hline Sepsis & $0 \%$ & $1 \%$ & $4.38(1.24-15.42)$ & $0.021 *$ \\
\hline Death & $0 \%$ & $3 \%$ & $9.59(2.90-31.64)$ & $<0.001 *$ \\
\hline Unplanned readmission & $2 \%$ & $4 \%$ & $1.80(1.00-3.24)$ & $0.049 *$ \\
\hline Discharge to IP facility & $55 \%$ & $67 \%$ & $1.68(1.39-2.02)$ & $<0.001 *$ \\
\hline
\end{tabular}

* Probability values $<0.05 ; \mathrm{OA}=$ osteoarthritis; FNF $=$ femoral neck fracture; OR $=$ odds ratio; $\mathrm{CI}=$ confidence interval; $\mathrm{DVT}=$ deep venous thrombosis; $\mathrm{PE}=$ pulmonary embolism; $\mathrm{IP}=$ inpatient.

suffer from human error as well as limited clinical information (specifically, we could not review radiographs or clinical charts). However, the interrater frequency of disagreement in recent NSQIP audits of its data is less than $2 \%$ [18]. Second, there may be residual bias that may have affected how the patients with femoral neck fractures were selected for THA rather than another treatment such as hemiarthroplasty. We attempted to control for this using a weighted propensity score model, but this can only control for observed variables. Additionally, recent evidence has shown that discharge to postacute care facilities is associated with numerous complications, including unplanned readmission, after hospital discharge [12]. These associations may have confounded some of our results. Third, data are limited to a relatively short (30-day) followup period. Currently proposed bundled payment models extend over 90-day episodes of care [7], and further study is warranted to assess longer-term outcomes between these two groups. We speculate that more complications would accumulate for the fracture group over a longer followup period. However, prior studies comparing cohorts of patients with femoral neck fracture and patients with OA have been limited to primarily in-hospital analyses [13, 17]. Finally, only THAs were analyzed, yet differences between hip arthroplasty cohorts that include patients receiving hemiarthroplasty for fracture would likely be more profound, because there is typically surgeon selection bias for performing hemiarthroplasty over THA in more infirm and more elderly patients with femoral neck fractures [2].

In 2013, Sassoon et al. [17] used a national discharge database from 1990 to 2007 to compare the in-hospital outcomes of unmatched patient cohorts for whom THA had been performed for OA or femoral neck fractures. They found that the fracture group had higher rates of perioperative mortality, pulmonary embolism, hematoma, and infection. Additionally, patients with fractures were more likely to be discharged to a rehabilitation facility than patients with OA. Similarly, a recent article using the National French Hospital Discharge Database performed a matched comparison of THA in patients with either OA or femoral neck fracture [13]. Their findings were very similar to those of the current study: patients with fractures were older and had more medical comorbidities than those undergoing elective THA for OA, but even after matching, 
there were higher complication rates in the patients with fractures than those with OA. Although the study was limited in that followup was only 72 hours after discharge, these consistent findings show the strong differences between these two patient groups undergoing THA.

We speculate that the observed differences between the matched cohorts may be partially attributable to preoperative medical optimization that patients with OA may receive before elective THA. Patients with femoral neck fractures receive THA in an urgent setting, in which the same degree of preoperative optimization cannot occur. The nearly 2-day delay from hospital admission to the operating room for patients with fractures compared with patients with OA implies the in-hospital burden required for preoperative medical management of patients with femoral neck fractures. Furthermore, there are likely patient-level factors and factors specific to the physiological response to hip fracture that were not captured in these data and that explain the observed differences between diagnoses, which persisted even after matching for comorbidities.

For all healthcare providers, it is important to consider the impact that fracture status imparts on the outcomes of THA when considering health policy initiatives. To address the rapid growth of healthcare spending, healthcare delivery in the United States is transitioning from a fee-forservice model to alternative reimbursement models such as bundled payments. Specifically for arthroplasty, the Centers for Medicare \& Medicaid Services will soon implement a comprehensive care model that will include a fixed reimbursement rate to cover all costs of care from the initial surgery and hospitalization and all associated care and recovery within 90 days of discharge [7]. This current study shows that, compared with patients with OA, patients with femoral neck fractures undergoing THA have significantly more complications, a longer length of hospital stay, and a higher rate of postdischarge to a rehabilitation center, which all amount to higher utilization of resources. Therefore, femoral neck fracture is a risk factor for numerous in-hospital complications and adverse events over at least a 30-day period of care.

From a health policy perspective, if a MS-DRGanchored episode of care reimbursement is not appropriately risk-stratified based on a patient's fracture status, hospitals that care for a high percentage of patients with hip fractures may be unfairly disadvantaged compared with hospitals that predominantly provide elective THA for OA. The latter hospitals may enjoy an economic advantage. Furthermore, target pricing for episodes of care is frequently defined by historical and regional averages that may not reflect currentday and hospital-specific practices. This is a particular concern for low-volume hospitals, which can be disproportionately impacted by outlier cases and changes in their case mix. Furthermore, reimbursement does not necessarily equal actual costs of care. Therefore, hospitals that treat a significant number of hip fractures face risk for both target prices that do not reflect their current case mix and also reimbursement that does not meet their costs.

Although THA is considered an excellent option for many patients with femoral neck fractures, reimbursement systems should provide additional resources to account for the more complicated patient care necessary to treat femoral neck fractures compared with OA. Ultimately, inclusion of femoral neck fractures in a bundled payment model may limit the available resources for all patients undergoing THA if appropriate risk stratification is not provided. It is possible that hospitals will ration the proportion of THA for patients with OA and patients with femoral neck fracture to account for the increased risk femoral neck fractures represent. Therefore, it will be prudent going forward for healthcare payers to differentiate patients undergoing THA and compensate for their care based on fracture status, either by using a separate grouping or modifying code, to maximize the availability of joint arthroplasty to all patients. The rationale for risk stratification based on fracture status extends to patients undergoing hemiarthroplasty as well, who are typically more frail than patients with fracture who receive THA [2].

THA to treat femoral neck fracture is associated with greater rates of complications, longer LOS, and increased frequency of discharge to postacute care inpatient facilities compared with elective THA for OA, implying greater utilization of healthcare resources. These differences persist despite matching of preoperative risk factors. Thus, healthcare payers should appropriately recognize the expected differences in resource utilization for THA in treating femoral neck fractures compared with OA. For example, the Centers for Medicare \& Medicaid Services has recently selected a simple risk stratification method that sets different target prices for MS-DRG 470 and 469anchored episodes of care for patients with fractures and those without fractures. Future studies should examine patient outcomes and costs after THA performed for a variety of diagnoses. These studies should examine various time intervals, including 90 days and 1 year after surgery. These data should be used to develop more robust and granular risk-stratification methods that might be applied to prospective payments in alternative reimbursement models.

Acknowledgments We thank Yuo-yu Lee MS, for reviewing the statistical methods used in this analysis.

\section{References}

1. Abboud JA, Patel RV, Booth RE Jr, Nazarian DG. Outcomes of total hip arthroplasty are similar for patients with displaced 
femoral neck fractures and osteoarthritis. Clin Orthop Relat Res. 2004;421:151-154.

2. Alolabi N, Alolabi B, Mundi R, Karanicolas PJ, Adachi JD, Bhandari M. Surgical preferences of patients at risk of hip fractures: hemiarthroplasty versus total hip arthroplasty. $B M C$ Musculoskelet Disord. 2011;12:289.

3. Baker RP, Squires B, Gargan MF, Bannister GC. Total hip arthroplasty and hemiarthroplasty in mobile, independent patients with a displaced intracapsular fracture of the femoral neck. A randomized, controlled trial. J Bone Joint Surg Am. 2006;88: 2583-2589.

4. Blomfeldt R, Tornkvist H, Ponzer S, Soderqvist A, Tidermark J. Comparison of internal fixation with total hip replacement for displaced femoral neck fractures. Randomized, controlled trial performed at four years. J Bone Joint Surg Am. 2005;87:16801688.

5. Brauer CA, Coca-Perraillon M, Cutler DM, Rosen AB. Incidence and mortality of hip fractures in the United States. JAMA. 2009;302:1573-1579.

6. Caliendo M. Some practical guidance for the implementation of propensity score matching. J Econ Surv. 2008;22:31-72.

7. Centers for Medicare \& Medicaid Services. Comprehensive Care for Joint Replacement Model. Available at: https://innovation. cms.gov/initiatives/cjr. Accessed November 28, 2015.

8. Chammout GK, Mukka SS, Carlsson T, Neander GF, Stark AW, Skoldenberg OG. Total hip replacement versus open reduction and internal fixation of displaced femoral neck fractures: a randomized long-term follow-up study. J Bone Joint Surg Am. 2012;94:1921-1928.

9. Gebhard JS, Amstutz HC, Zinar DM, Dorey FJ. A comparison of total hip arthroplasty and hemiarthroplasty for treatment of acute fracture of the femoral neck. Clin Orthop Relat Res. 1992;282: 123-131.

10. Gjertsen JE, Vinje T, Lie SA, et al. Patient satisfaction, pain, and quality of life 4 months after displaced femoral neck fractures: a comparison of 663 fractures treated with internal fixation and 906 with bipolar hemiarthroplasty reported to the Norwegian Hip Fracture Register. Acta Orthop. 2008;79:594-601.

11. Healy WL, Iorio R. Total hip arthroplasty: optimal treatment for displaced femoral neck fractures in elderly patients. Clin Orthop Relat Res. 2004;429:43-48.

12. Keswani A, Tasi MC, Fields A, Lovy AJ, Moucha CS, Bozic KJ. Discharge destination after total joint arthroplasty: an analysis of postdischarge outcomes, placement risk factors, and recent trends. J Arthroplasty. 2016 Jan 20. [Epub ahead of print].

13. Le Manach Y, Collins G, Bhandari M, Bessissow A, Boddaert J, Khiami F, Chaudhry H, De Beer J, Riou B, Landais P, Winemaker M, Boudemaghe T, Devereaux PJ. Outcomes after hip fracture surgery compared with elective total hip replacement. JAMA. 2015;314:1159-1166.

14. Lombardi B, Paci M, Nannetti L, Moretti S, Maritato M, Benelli G. Total hip arthroplasty after hip fracture or osteoarthritis: are there differences in characteristics and outcomes in the early rehabilitative stage? Orthop Nurs. 2014;33:43-47.

15. Macaulay W, Nellans KW, Garvin KL, Iorio R, Healy WL, Rosenwasser MP, DFACTO Consortium. Prospective randomized clinical trial comparing hemiarthroplasty to total hip arthroplasty in the treatment of displaced femoral neck fractures: winner of the Dorr Award. J Arthroplasty. 2008;23(Suppl 1):2-8.

16. Miller BJ, Cai X, Cram P. Mortality rates are similar after hip fractures for rural and urban patients. Clin Orthop Relat Res. 2012;470:1763-1770.

17. Sassoon A, D’Apuzzo M, Sems S, Cass J, Mabry T. Total hip arthroplasty for femoral neck fracture: comparing in-hospital mortality, complications, and disposition to an elective patient population. J Arthroplasty. 2013;28:1659-1662.

18. Shiloach M, Frencher SK Jr, Steeger JE, Rowell KS, Bartzokis K, Tomeh MG, Richards KE, Ko CY, Hall BL. Toward robust information: data quality and inter-rater reliability in the American College of Surgeons National Surgical Quality Improvement Program. J Am Coll Surg. 2010;210:6-16.

19. Sierra RJ, Cabanela ME. Conversion of failed hip hemiarthroplasties after femoral neck fractures. Clin Orthop Relat Res. 2002;399:129-139.

20. Wani IH, Sharma S, Latoo I, Salaria AQ, Farooq M, Jan M. Primary total hip arthroplasty versus internal fixation in displaced fracture of femoral neck in sexa- and septuagenarians. J Orthop Traumatol. 2014;15:209-214.

21. Yu L, Wang Y, Chen J. Total hip arthroplasty versus hemiarthroplasty for displaced femoral neck fractures: meta-analysis of randomized trials. Clin Orthop Relat Res. 2012;470:22352243.

22. Zi-Sheng A, You-Shui G, Zhi-Zhen J, Ting Y, Chang-Qing Z. Hemiarthroplasty vs primary total hip arthroplasty for displaced fractures of the femoral neck in the elderly: a meta-analysis. $J$ Arthroplasty. 2012;27:583-590. 